\title{
SOME RESULTS FOR THE QUADRATIC ANALYSIS OF GAUSSIAN PROCESSES AND APPLICATIONS
}

\author{
J.-L. SOLER
}

Université Scientifique et Médicale de Grenoble, Grenoble, France

\section{Introduction}

This paper is concerned with the study of the random variable $X \otimes X$, when $X$ is an infinitedimensional Gaussian random variable. It is shown to be a sufficient statistic on Gaussian statistical spaces with unknown variance by means of the theory of Generalized Exponential Families introduced in [13]. Then, the calculus of its characteristic functional when $X$ lies in a separable Fréchet space, provides an infinite dimensional non-central Wishart distribution as anounced in [14]. The characteristic functions of quadratic forms of certain non-central Gaussian real random functions are derived and then they are used in statistical applications to the classical theory of goodness of fit tests and to the test of significance of the mean function of an observed Gaussian process with known covariance function, and other related tests.

\section{Gaussian statistical spaces with unknown variance and generalized exponential families}

Let us first recall some definitions and generalities from [12], [13], [15] and then we shall state the main theorem of this section which justifies the study.

A vector statistical space (V.S.S.) will be a triplet $(E, \mathscr{A}, \mathscr{P})$, that is, the mathematical model associated with a statistical experiment where $E$ is a vector space (over $\boldsymbol{R}$ ) representing the space of all the possible (vector) observations $x, \mathscr{A}$ is the $\sigma$-field of subsets of $E$ generated by the observable events and $\mathscr{P}$ is a family of hypotheses concerning the probability distribution of the observed random variable $X$ in $(E, \mathscr{A})$. Here, we shall consider especially infinite dimensional spaces $E, X$ being the natural random variable associated with a stochastic process whose sample paths are in $E$ (random functions, random measures, etc.).

For the sake of generality and to avoid the consideration of any a priori topology on $E$ we shall assume for the $\sigma$-field $\mathscr{A}$ to be such that the mappings: $(\lambda, x) \mapsto \lambda x$ 
of $\boldsymbol{R} \times E$ onto $E$ and $(x, y) \mapsto x+y$ of $E \times E$ onto $E$ are measurable when $\boldsymbol{R}$ is endowed with its Borel $\sigma$-field. In this case, the pair $(E, \mathscr{A})$ will be called a measurable vector space (M.V.S.).

Let $(E, \mathscr{A})^{m}$ denote the dual of the M.V.S. $(E, \mathscr{A})$, that is, the vector space of all the $\mathscr{A}$-measurable linear forms on $E$. We shall systematically refer ourself to the canonical duality $\left\langle E,(E, \mathscr{A})^{m}\right\rangle$ and assuming it to be separated we shall call the pair $(E, \mathscr{A})$ a separated M.V.S. (S.M.V.S.). In this case, the sub $\sigma$-field $\mathscr{C}\left(E,(E, \mathscr{A})^{m}\right)$ of $\mathscr{A}$ generated by the elements of $(E, \mathscr{A})^{m}$, or also by the cylinder subsets of $E$ w.r. to this duality, will be called the weakened $\sigma$-field of $\mathscr{A}$, and we have' $\left(E, \mathscr{C}\left(E,(E, \mathscr{A})^{m}\right)\right)^{m}=(E, \mathscr{A})^{m}$. A characterization of this dual is given in [12] for a wide class of S.M.V.S. $(E, \mathscr{A})$; especially, it is shown in [15] that every Borel linear form defined on a separable Fréchet space is continuous, which may be written $(E, \mathscr{B}(E))^{m} \equiv E^{\prime}$, however it is well known that $\mathscr{C}\left(E, E^{\prime}\right)=\mathscr{B}(E)$ in this assumption.

Now, if $P$ is any probability on a S.M.V.S. $(E, \mathscr{A})$ and if $\mathscr{A}$ contains all the $P$-negligible subsets of $E$, the triplet $(E, \mathscr{A}, P)$ will be called a probability vector space (P.V.S.)

Derinition 1.1. The dual of the P.V.S. $(E, \mathscr{A}, P)$ is the vector space $L(E, \mathscr{A}, P)$ of the equivalence classes of the elements of the vector space $\mathscr{L}(E, \mathscr{A}, P)$ of all the $\mathscr{A}$-measurable real functionals which are defined and are linear on a vector subspace of probability 1 of $E$. These elements will be called linear real random variables (1.r.r.v.).

Such functionals have been already considered by several authors (see e.g. [9]). Evidently, the quotient space $(E, \mathscr{A})^{m} / P$ is contained in $\mathscr{L}(E, \mathscr{A}, P) / P=$ $L(E, \mathscr{A}, P)$; a detailed study of this space is made in [15]. The functional $\Phi_{P}$ [resp. $L_{P}$ ] defined in $L(E, \mathscr{A}, P)$ by: $\Phi_{P}(\xi)=\int_{E} e^{i \xi} d P$ for all $\xi \in L(E, \mathscr{A}, P)$ [resp. $L_{P}(\xi)=\int_{E} e^{\xi} d P$ for $\xi \in D(E, \mathscr{A}, P)$ which is the convex subset of $L(E, \mathscr{A}, P)$ where this integral is finite] is called the Fourier transform [resp. the real Laplace transform] of the P.V.S. and may be regarded as the extension of the Fourier transform $\varphi_{P}$ [resp. the real Laplace transform $l_{P}$ ] of the probability $P$ on $(E, \mathscr{A})$ which is defined in $(E, \mathscr{A})^{m}$ by: $\varphi_{P}(y)=\int_{E} e^{l(x, y)} d P(x)$ for all $y \in(E, \mathscr{A})^{m}$ [resp. $l_{P}(y)$ $=\int_{E} e^{\langle x, y\rangle} d P(x)$ for $y \in(E, \mathscr{A})^{m}$ for which this integral is finite] since the value of these functions at $y$ depends only on its equivalence class in $(E, \mathscr{A})^{m} / P$. families.

Let us state now the definition of a generalized notion of that of exponential

DefrNItIoN 1.2. A V.S.S. $(E, \mathscr{A}, \mathscr{P})$ is called of canonical exponential type (C.E.T.) iff:

(i) $\mathscr{P}$ is a family of equivalent probabilities on $(E, \mathscr{A})$;

(ii) $\mathscr{A}$ contains all the P-negligible subsets of $E, P \in \mathscr{P}$; (iii) for some fixed probability $P^{*} \in \mathscr{P}$,

$$
\left\{\log d P / d P^{*} ; P \in \mathscr{P}\right\} \subset L\left(E, \mathscr{A}, P^{*}\right) \oplus \boldsymbol{R} .
$$

A statistical space $(\mathscr{X}, \mathscr{F}, \mathscr{P})$ is of exponential type (E.T.) iff there exists a sufficient statistic $T$ with values in a S.M.V.S. $(E, \mathscr{A})$ whose image statistical space $\left(E, \mathscr{A}, \mathscr{P}_{T}=\left\{P \circ T^{-1} ; P \in \mathscr{P}\right\}\right)$ is of C.E.T.

Clearly, this definition does not depend on $P^{*}$ in $\mathscr{P}$. It is equivalent to the following parametrical one:

Definition 1.3. A statistical space $\left(\mathscr{X}, \mathscr{F}, \mathscr{P}=\left\{P_{\theta} ; \theta \in \Theta\right\}\right)$ is of E.T. w.r. to the sufficient statistic $T$ with values in the S.M.V.S. $(E, \mathscr{A})$ iff:

(i) $\Theta$ is a subset, containing the origin, of the convex $D\left(E, \mathscr{A}, P_{0} \circ T^{-1}\right) \subset$ $L\left(E, \mathscr{A}, P_{0} \circ T^{-1}\right)$ (which is the domain of definition of the real Laplace transform $L_{P_{0} \circ T^{-1}}$ of the P.V.S. $\left(E, \mathscr{A}, P_{0} \circ T^{-1}\right)$ );

(ii) the $P_{\theta}, \theta \in \Theta$, are equivalent on the sub- $\sigma$-field of $\mathscr{F}$ generated by $T$ (which is assumed to contain the negligible subsets of $\mathscr{X}$ w.r. to the restrictions of the $P_{0}$ on it);

(iii) for every $\theta \in \Theta$

$$
\frac{d P_{\theta}}{d P_{0}}=\frac{e^{\theta \circ T}}{L_{P_{0} \circ T^{-1}}(\theta)}
$$

Since actually we are concerned with Gaussian V.S.S., let $P_{m}$ be the Gaussian probability on $(E, \mathscr{A})$ with mean $m \in E$ and "variance" the non-negative quadratic form $Q_{0}$ on $(E, \mathscr{A})^{m}$, i.e.

$$
\varphi_{\boldsymbol{P}_{m}}(y)=\exp \left\{i\langle m, y\rangle-\frac{1}{2} Q_{0}(y)\right\}, \quad y \in(E, \mathscr{A})^{m} .
$$

Let $H\left(Q_{0}\right)$ denote the closure of $(E, \mathscr{A})^{m} / P_{0}$ in $L_{2}\left(E, \mathscr{A}, P_{0}\right)$ and let $\mathscr{H}\left(Q_{0}\right)$ denote the Hilbert space of functionals on $(E, \mathscr{A})^{m} / P_{0}$ which is in correspondence with $H\left(Q_{0}\right)$ by the isometry: $Z \mapsto E_{P_{0}}(Z \cdot Y)=h_{Z}(Y), Y \in(E, \mathscr{A})^{m} / P, Z \in H\left(Q_{0}\right)$.

Proposirion 1.1. If $\mathscr{H}\left(Q_{0}\right) \subset E$, then $L\left(E, \bar{A}, P_{0}\right) \equiv H\left(Q_{0}\right)$ and the Gaussian statistical space with unknown mean $\left(E, \overline{\mathscr{A}},\left\{P_{m} ; m \in \mathscr{H}\left(Q_{0}\right)\right\}\right)$ is of C.E.T. ( $\overline{\mathscr{A}}$ denotes the completion of $\mathscr{A}$ by the $P_{0}$-negligible subsets of $E$ ).

The proof is obvious and results from the density of the equivalent probabilities $P_{m}$ w.r. to $P_{0}$ when $m \in \mathscr{H}\left(Q_{0}\right)$.

Proposirion 1.2. With the same assumptions, the Gaussian statistical space with unknown variance $\left(E, \bar{A}, \mathscr{P}_{Q_{0}}\right)$, where $\mathscr{P}_{Q_{0}}$ denotes the set of all the centered Gaussian probabilities on $(E, \mathscr{A})$ which are equivalent to $P_{0}$, is of E.T. w.r. to the suffcient statistic $\dot{\chi}=\chi_{E}: x \mapsto x \otimes x$ of $E$ into the tensor product space $E \otimes E$.

The proof results from the density of any $P_{Q} \in \mathscr{P}_{Q_{0}}$ w.r. to $P_{0}=\left(P_{Q_{0}}\right)$ which is of the form: $d P_{Q} / d P_{0}=\exp \left(\zeta_{Q}\right) / E_{P_{0}}\left[\exp \left(\zeta_{Q}\right)\right]$ where $\zeta_{Q}$ is a unique element of the Hilbert space $H\left(Q_{0}\right) \tilde{\odot} H\left(Q_{0}\right)$ which is the completed symmetric tensor product of $H\left(Q_{0}\right)$ by itself (see [7]), and from 
LEMMA 1.1. If $E_{\chi}=\operatorname{sp}(\chi(E))$ in $E \otimes E$ and if $\overline{\mathscr{A}}_{\chi}$ is the greatest $\sigma$-field on $E_{\chi}$ which yields $\chi$ measurable, then, the Hilbert space $H\left(Q_{0}\right) \odot H\left(Q_{0}\right)$ is isomorphic to a vector subspace of the dual $L\left(E_{\chi}, \overline{\mathscr{A}}_{\chi}, P_{0} \circ \chi^{-1}\right)$ and every element $\zeta \in H\left(Q_{0}\right) \tilde{\odot}$ $\tilde{\odot} H\left(Q_{0}\right)$ can be written as $\zeta=\xi \circ \chi$ where $\xi$ is a unique l.r.r.v. on the P.V.S. $\left(E_{\chi}, \overline{\mathscr{A}}_{\chi}\right.$, $P_{0} \circ \chi^{-1}$ ), (see [13], [15]).

Thus, $d P_{Q} / d P_{0}=\exp \left(\xi_{Q} \circ \chi\right) / L_{P_{0} \circ \chi^{-1}}\left(\xi_{Q}\right)$ which shows that be statistical space $\left(E, \bar{A}, \mathscr{P}_{Q_{0}}\right)$ if of E.T. w.r. to $\chi$.

The description of the C.E.T. V.S.S. $\left(E_{\chi}, \overline{\mathscr{A}}_{x},\left\{P_{Q} \circ \chi^{-1} ; P_{Q} \in P_{Q_{0}}\right\}\right)$ induced by $\chi$ justifies the study of the probability $P_{Q} \circ \chi^{-1}$ which is the distribution of the random variable $X \otimes X$ when $X$ is Gaussian.

\section{The canonical non-central Wishart cylindrical probability}

Let $E$ be a real or complex vector space and let $u$ be a linear mapping of $E$ into itself. For every positive integer $n, u^{n} \otimes, u^{n} \odot, u^{n \wedge}$ will denote respectively the $n$th ordinary, symmetric, exterior, tensor powers of $u$, which are respectively linear mappings of the ordinary, symmetric, exterior, tensor product spaces $E^{n \otimes}, E^{n} \odot$, $E^{n \wedge}$ into themselves. If $u$ is of finite rank, let us set $\alpha_{n}(u)=\operatorname{Trace}\left(u^{n \wedge}\right), \alpha_{0}(u)=1$ and $\operatorname{det}(\mathbf{1}+u)=\sum_{n \geqslant 0} \alpha_{n}(u)$ pointing out that the summation is finite and that it coincides with the determinant of the linear mapping $(\mathbf{1}+u)$ if $E$ is finite dimensional (see [4]).

Now, let $H$ be a real or complex Hilbert space with scalar product $\langle\cdot, \cdot\rangle_{H}$. (If $H$ is real we shall denote by $H_{c}$ its complexification $H+i H$, in case of need.)

Let $H \tilde{\odot} H$ denote the Hilbert space obtained by completing the separated prehilbertian space $H \odot H$, for the scalar product $\left\langle x \otimes x^{\prime}, y \otimes y^{\prime}\right\rangle_{H \odot H}=$ $\langle x, y\rangle_{H} \cdot\left\langle x^{\prime}, y^{\prime}\right\rangle_{H}$.

Let $H \widehat{\odot} H$ denote the Banach space obtained by completing the symmetric algebraic tensor product $H \odot H$, endowed with the trace-norm, which is called the completed projective symmetric tensor product of $H$ by itself (see [4], [11]). As usual, the spaces $H \odot H, H \tilde{\odot} H, H \hat{\odot} H$ will be identified respectively with the spaces of continuous self-adjoint of finite rank, or Hilbert-Schmidt, or nuclear operators in $H$.

LEMMA 2:1 (see [4]), For every positive integer $n, u \mapsto \alpha_{n}(u)$ is a continuous form on the normed space $H \odot H$ (for the trace-norm) and it admits a continuous extension to $H \odot \hat{\odot} H$. Moreover, $\sum_{n \geqslant 0} \alpha_{n}(u)$ is an absolutely convergent series and it is still denoted by: $\operatorname{det}(\mathbf{1}+u)$ when $u \in H \widehat{\odot} H$. The operator $\mathbf{1}+u$ is invertible iff: $\operatorname{det}(\mathbf{1}+u) \neq 0$.

The mapping $z \mapsto \operatorname{det}(1-z u)$ defined on the scalar field of $H$, is called the Fredholm determinant of $u \in H \hat{\odot} H$.
Let us recall that if $\langle E, F\rangle$ is a separated duality, a cylindrical probability $\mu$ on $E$ w.r. to this duality is a projective system of finite dimensional P.V.S.: $(E / M$, $\left.\mathscr{B}(E / M), \mu_{M}\right)_{M \in \mathscr{M}(E, F)}$ where $\mathscr{M}(E, F)$ denotes the filter base of the subspaces of $E$ finite codimension. Such a cylindrical probability $\mu=\left(\mu_{M}\right)_{M \in \mathscr{M}(E, F)}$ is uniquely determined by its Fourier transform which is defined on $F$ by: $\varphi_{\mu}(y)=\int_{R} e^{i t} d \mu_{y}(t)$, $y \in F$, where $\mu_{y}$ denotes the induced probability on $(\boldsymbol{R}, \mathscr{B}(\boldsymbol{R}))$ by the linear weak continuous mapping: $x \mapsto\langle x, y\rangle$ on $E$.

It does not necessarily define a probability on the S.M.V.S. $(E, \mathscr{C}(E, F))$.

If $E$ is a separable real Hilbert space, say $H$, a cylindrical probability $\mu \cdot$ on $H$ (w.r. to the canonical duality $\langle H, H\rangle_{H}$ ) reduces to the projective system of finite dimensional P.V.S.: $\left(V, \mathscr{B}(V), \mu_{V}\right)_{V_{\in} \mathscr{F}_{(H)}}$, where $\mathscr{F}(H)$ denotes the family of all the finite dimensional subspaces of $H$.

LEMMA 2.2. Let $\mu$ be a cylindrical probability on a real separable Hilbert space $H$. Then the "quadratic mapping": $\chi_{H}: x \mapsto x \otimes x, x \in H$, induces a cylindrical probability on $H \odot H$ w.r. to the canonical duality $\langle H \odot H, H \odot H\rangle_{H \odot H}$ of this separated prehilbertian space. It will be called the "quadratic image of $\mu$ " and it will be denoted $b y: \chi_{H}(\mu)$.

Proof. We have to construct a projective system $\left(K, \mathscr{B}(K), \nu_{K}\right)_{K \in \mathscr{F}(H \odot H)}$ of finite-dimensional P.V.S. uniquely determined by $\mu$ and $\chi_{H}$. First, we observe that the family

$$
\left(V \odot V, \mathscr{B}(V \odot V), \chi_{V}\left(\mu_{V}\right)\right)_{V \in \mathscr{F}(H)}
$$

is a projective system of finite-dimensional P.V.S., since if $V \supset W$ in $\mathscr{F}(H), V \odot V$ $\supset W \odot W$ in $\mathscr{F}(H \odot H)$, and denoting by $P_{V, W}$ the orthogonal projector of $V$ onto $W$ and $P_{V \odot V, W \odot W}$ the corresponding one of $V \odot V$ onto $W \odot W$ we have

$$
P_{V \odot V, W \odot W}=P_{V, W}^{2 \odot} \text { and } P_{V, W}^{2 \odot} \circ \chi_{V}=\chi_{W} \circ P_{V, W} .
$$

It follows that $\chi_{W}\left(\mu_{W}\right)=\chi_{W} \circ P_{V, W}\left(\mu_{V}\right)=P_{V, W}^{2 \odot} \circ \chi_{V}\left(\mu_{V}\right)=P_{V \odot V, W \odot W}\left(\chi_{V}\left(\mu_{V}\right)\right)$, which is the necessary relation of compatibility.

Now, let $K \in \mathscr{F}(H \odot H)$. If $K=V \odot V$ for some $V \in \mathscr{F}(H)$, then set: $\nu_{K}=\chi_{V}\left(\mu_{V}\right)$ if not, setting: $V_{K}=\operatorname{sp}\left\{\chi_{H}^{-1}(K)\right\} \subset H, V_{K} \odot V_{K}$ is easily seen to be the smallest element of $\mathscr{F}(H \odot H)$ having the preceding form and containing $K$, then set: $\nu_{K}$ $=P_{V_{K} \odot V_{K}, K}\left(v_{V_{K}}\right)$. It is now easy to verify that the family

$$
\left(K, \mathscr{B}(K), v_{K}\right)_{K \in \mathscr{F}(H \odot H)}
$$

is a projective system of finite-dimensional P.V.S. Clearly, it is uniquely determined by $\mu$ and $\chi_{H}$ by construction.

Let $m$ be any fixed element of a real Hilbert space $H$ and let us denote by $\mu_{m}$ the non-central canonical Gaussian cylindrical probability on $H$, whose Fourier transform is: $\varphi_{\mu_{m}}(x)=\exp \left\{i\langle m, x\rangle_{H}-\frac{1}{2}\|x\|_{H}^{2}\right\}, x \in H$.

PRoposition 2.1. The Fourier transform of the quadratic image of $\mu_{m}$ is: (2.1) $\varphi_{z_{n}\left(\mu_{m}\right)}(u)=\operatorname{det}(\mathbf{1}-2 i u)^{-1 / 2} \exp \left\{\dot{i}\left\langle m,(1-2 i u)^{-1} \circ u(m)\right\rangle_{H_{c}}\right\}, \quad u \in H \odot H$. 
Proof. Since $H \odot H=\bigcup_{K \in \mathscr{F}(H \odot H)} K$, it is sufficient that (2.1) be true on every finite-dimensional subspace of $H \odot H$, and by the construction of $\chi_{H}\left(\mu_{m}\right)$ we may restrict ourself to the ones of the form $V \odot V$ for $V \in \mathscr{F}(H)$. Now, if $V \in \mathscr{F}(H)$, denoting by $P_{V}$ the orthogonal projector of $H$ onto $V, \mu_{m_{V}}$ is the Gaussian probability on $(V, \mathscr{B}(V))$ with mean $P_{V}(m)$ and "variance" the unit quadratic form on $V$. Thus, the calculus of the Fourier transform of $\chi_{V}\left(\mu_{m_{q}}\right)$ results directly from the following classical result:

LEMMA 2.3 (Wishart-James), Let $X$ be a Gaussian random variable on $\left(\boldsymbol{R}^{k}, \mathscr{B}\left(\boldsymbol{R}^{k}\right)\right)$ with mean $a \in \boldsymbol{R}^{k}$ and covariance matrix $A$. Then the characteristic function of the random matrix: $X \cdot{ }^{t} X$ in the Hilbert space $S_{k}$ of symmetric matrices of order $k$ for the scalar product $\langle A, B\rangle_{S_{k}}=\operatorname{Trace}(A \cdot B)$ is:

$\varphi_{X^{t}{ }_{X}}(U)=\operatorname{det}(1-2 i U \Lambda)^{-1 / 2} \exp \left\{i \cdot \operatorname{Trace}\left[a \cdot{ }^{t} a \cdot U \cdot(1-2 i U \Lambda)^{-1}\right]\right\}, \quad U \in S_{k}$.

And $E\left(X \cdot{ }^{t} X\right)=\left(a \cdot{ }^{t} a+\Lambda\right) .\left({ }^{t} X\right.$ denotes the transposed vector of $\left.X\right)$.

Indeed, assuming for the dimension of $V$ to be $k$, by the choice of an orthogonal basis in $V, V$ becomes isometric to $R^{k}$ and $V \odot V$ becomes isometric to $S_{k}$. Taking $a=P_{V}(m)$ and $\Lambda=1$ we obtain:

$$
\varphi_{\chi_{V}\left(\mu_{m_{V}}\right)}(u)=\operatorname{det}(1-2 i u)^{-1 / 2} \exp \left\{i \cdot \operatorname{Trace}\left[P_{V}(m) \otimes P_{V}(m) \circ u \circ(1-2 i u)^{-1}\right]\right\},
$$$$
u \in V \odot V \text {, }
$$

where $\operatorname{det}(1-2 i u)$ is the value at $2 i$ of the Fredholm determinant of the finite rank operator $u$ in $V$ corresponding to the matrix $U$, which coincides with the determinant of the matrix $(1-2 i U) \in S_{k}+i S_{k}$.

Finally, since $P_{V}(m) \otimes P_{V}(m)=P_{V}^{2 \odot}(m \otimes m)=P_{V \odot V}(m \otimes m)$, we have

$\operatorname{Trace}\left[P_{V}(m) \otimes P_{V}(m) \circ u \circ(1-2 i u)^{-1}\right]=\left\langle P_{V}(m) \otimes P_{V}(m), u \circ(1-2 i u)^{-1}\right\rangle_{H_{c} \odot H_{c}}$

$$
=\left\langle m \otimes m, u \circ(1-2 i u)^{-1}\right\rangle_{H_{c} \otimes H_{c}}
$$$$
=\left\langle m,(1-2 i u)^{-1} \circ u(m)\right\rangle_{H_{c}}
$$

which implies that (2.1) is true for every $u \in V \odot V, V \in \mathscr{F}(H)$ since in this case, $\varphi_{\chi_{B}\left(\mu_{m}\right)}(u)=\varphi_{\chi_{V}\left(\mu_{m_{V}}\right)}(u)$.

Defrinition 2.1. The quadratic image $\chi_{H}\left(\mu_{m}\right)$ will be called the canonical noncentral Wishart cyclindrical probability with one degree of freedom and it will be denoted by $w_{H}\left(1, m,\|\cdot\|_{H}\right)$.

Now, we point out that the complex functional defined by (2.1) is continuous on $H \odot H$ for the projective topology. This results from the Fredholm theory [4]. Thus, by Lemma 2.1 there exists a unique continuous extension of this functional

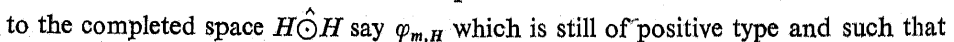
$\varphi_{m, H}(0)=1$. By a classical result we can state then:

Proposition 2.2. There exists a unique cylindrical probability on $H \odot H$ w.r. to duality $\langle H \odot H, H \odot H\rangle$ which is the restriction to these spaces of the duality $\langle H \tilde{\odot} H, H \tilde{\odot} H\rangle_{H \tilde{\odot} \boldsymbol{H}}$ provided by the Hilbertian structure of $H \odot \tilde{} H$, whose Fourier transform is:

$$
\varphi_{m, H}(u)=\operatorname{det}(1-2 i u)^{-1 / 2} \exp \left\{i\left\langle m,(1-2 i u)^{-1} \circ u(m)\right\rangle_{H_{c}}\right\}, \quad u \in H \hat{\odot} H,
$$

and the restriction of this functional to the space $H \odot H$ coincides with the Fourier transform of the quadratic image of the non-central canonical Gaussian cylindrical probability on $H$.

\section{The non-central Wishart distribution associated with separable Fréchet space valued Gaussian random variables}

This section deals with the study of an infinite-dimensional extension, in a quite general case, of the non-central Wishart distribution based on the results of Section 2.

Proposition 3.1. Let $E$ be a separable Fréchẹt space with Borel $\sigma$-field $\mathscr{B}(E)$ and topological dual $E^{\prime}$. Let $E \odot E$ [resp. $\left.E^{\prime} \odot E^{\prime}\right]$ be the projective symmetric tensor product of $E$ by itself [resp. of $E^{\prime}$ (endowed with its strong topology) by itself]. $\mathscr{B}(E \odot E)$ will denote the Borel $\sigma$-field of the topological space $E \odot E$ and $E^{\prime} \hat{\odot} E^{\prime}$ will denote the completion of the topological space $E^{\prime} \odot E^{\prime}$.

Let $N_{E}(0, Q)$ be the Gaussian probability on $(E, \mathscr{B}(E))$ with mean 0 and variance the non-negative quadratic form $Q$ on $E^{\prime}$.

Then there exists a separable Hilbert space $\mathscr{H}(Q) \subset E$ with continuous canonical injection and for every $m \in \mathscr{H}(Q)$, there exists a unique probability on $(E \odot E, \mathscr{B}(E \odot E))$ whose Fourier transform $\varphi$ is such that for every $t \in E^{\prime} \hat{\odot} E^{\prime}$,

(3.1) $\varphi(t)=\operatorname{det}\left(1-2 i \eta_{Q}(t)\right)^{-1 / 2} \exp \left\{i\left\langle m,\left(1-2 i \eta_{Q}(t)\right)^{-1} \circ \eta_{Q}(t)(m)\right\rangle_{\mathscr{N}(O)}\right\}$ where $\eta_{Q}$ is a continuous linear mapping of $E^{\prime} \hat{\odot} E^{\prime}$ into $\mathscr{H}(Q) \hat{\odot} \mathscr{H}(Q)$ which is uniquely determined by $Q$.

Moreover, if $X$ is a random variable with values in $(E, \mathscr{B}(E))$ of the form $m+X_{0}$ where $X_{0}$ has the $N_{E}(0, Q)$ distribution, equation (3.1) determines the characteristic functional of the random variable $X \otimes X$ with values in $(E \odot E, \mathscr{B}(E \odot E))$.

We shall denote by $W_{E}(1, m, Q)$ the distribution of $X \otimes X$ which is so characterized, and we shall call it the "Wishart distribution with one degree of freedom with parameters $m$ and $Q$ associated with the non-central Gaussian distribution $N_{B}(m, Q)$ on $(E, \mathscr{B}(E))$ ". The non-central Wishart distribution $W_{E}\left(n, m_{1}, \ldots, m_{n}, Q\right)$ will easily be defined by convolution.

Proof. We have already pointed out that with our assumptions, $\mathscr{B}(E)=\mathscr{C}\left(E, E^{\prime}\right)$ and $(E, \mathscr{B}(E))^{m}=E^{\prime}$. Thus,

$$
\varphi_{N_{E}(0, Q)}\left(x^{\prime}\right)=\exp \left\{-\frac{1}{2} Q\left(x^{\prime}\right)\right\}, \quad x^{\prime} \in E^{\prime} .
$$

Let us recall now an important theorem of the abstract Wiener spaces theory (see [8]): "with the assumptions of the proposition, there exists a separable Hilbert space $\mathscr{H}(Q)$ which is uniquely determined by $Q$ such that the canonical injection $j$ 
of $\mathscr{H}(Q)$ into $E$ is continuous and the image by $j$ of the canonical central Gaussian cylindrical probability $\mu_{0}$ on $\mathscr{H}(Q)$ is the Gaussian cylindrical probability on $E$ w.r. to the duality $\left\langle E, E^{\prime}\right\rangle$ associated with the Gaussian probability $N_{E}(0, Q)$ on $(E, \mathscr{B}(E))$ ". In other terms, $j$ "transforms" $\mu_{0}$ in a true probability on $(E, \mathscr{C}(E, F))$.

Now let $m \in \mathscr{H}(Q)$ and let $\mu_{m}$ be the non-central canonical Gaussian cylindrical probability on $\mathscr{H}(Q)$ as defined in Section 2. The continuous mapping $j$ is also continuous for the weak topologies $\sigma(\mathscr{H}(Q), \mathscr{H}(Q))$ and $\sigma\left(E, E^{\prime}\right)$ and the image of $\mu_{m}$ by $j$ is the non-central Gaussian probability $N_{E}(m, Q)$ and denoting by ${ }^{j} j$ the conjugate map of $j$ we have,

$$
\begin{aligned}
\varphi_{J\left(\mu_{m}\right)\left(x^{\prime}\right)} & \left.=\varphi_{\mu_{m}}{ }^{(t j} j\left(x^{\prime}\right)\right)=\exp \left\{i\left\langle m,{ }^{t} j\left(x^{\prime}\right)\right\rangle_{\mathscr{H}(Q)}-\frac{1}{2} \mid\left\|^{t} j\left(x^{\prime}\right)\right\|_{\mathscr{P}(Q)}^{2}\right\}, \quad x^{\prime} \in E^{\prime} \\
& =\exp \left\{i\left\langle m, x^{\prime}\right\rangle-\frac{1}{2} Q\left(x^{\prime}\right)\right\}, \quad x^{\prime} \in E^{\prime} .
\end{aligned}
$$

Setting $\chi_{E}: x \mapsto x \otimes x, x \in E$, we then have that $\chi_{E} \circ j\left(\mu_{m}\right)=\chi_{E}\left(N_{R}(m, Q)\right)$ is a probability on the S.M.V.S.: $\left(E \odot E, \mathscr{C}\left(E \odot E, E^{\prime} \odot E^{\prime}\right)\right)$. But, by: $\chi_{E} \circ j\left(\mu_{m}\right)=j^{2 \odot}$ 。 $\circ \chi \mathscr{H}(Q)\left(\mu_{m}\right)$, we have

$$
\varphi_{X_{E}\left(N_{E}(m, Q)\right)}=\varphi_{\left.X_{\mathscr{H}}(Q)\right)^{\left(\mu_{m}\right)}}{ }^{\circ}\left(j^{2} \odot\right) .
$$

And using (2.1), we have, for every $t \in E^{\prime} \odot E^{\prime}$,

(3.2) $\varphi_{x_{E}\left(N_{E}(m, Q)\right)}(t)$

$$
=\operatorname{det}\left(\mathbf{1}-2 i \cdot{ }^{t} j^{2} \odot(t)\right)^{-1 / 2} \exp \left\{i\left\langle m,\left(\mathbf{1}+2 i \cdot t^{2} \odot(t)\right)^{-1} \cdot{ }^{t} j^{2} \odot(t)(m)\right\rangle_{\mathscr{H}}\left(Q_{)}\right\} .\right.
$$

Now it is quite easy to prove (see [15]) that

$$
\mathscr{C}\left(E \odot E, E^{\prime} \odot E^{\prime}\right)=\mathscr{C}\left(E \odot E, E^{\prime} \hat{\odot} E^{\prime}\right)=\mathscr{B}(E \odot E) .
$$

Thus, (3.2) characterizes a unique Borel probability, i.e. $\chi_{E}\left(N_{E}(m, Q)\right)$ on $(E \odot E$, $\mathscr{B}(E \odot E))$.

But it is interesting in the applications to have an explicit expression of the Fourier transform of this probability on the greatest subspace of the topological dual $(E \odot E)^{\prime}$

Here, ${ }^{t}\left(j^{2 \odot} \odot\right)=\left({ }^{t}\right)^{2 \odot}$ and ${ }^{t} j$ is a weakly and strongly continuous mapping of $E^{\prime}$ into $\mathscr{H}(Q)$ (see [11]), so that ${ }^{t} j^{2} \odot$ is a continuous linear mapping of the projective symmetric tensor product $E^{\prime} \odot E^{\prime}$ into the corresponding one $\mathscr{H}(Q) \odot \mathscr{H}(Q)$ for the strong topologies on $E^{\prime}$ and $\mathscr{H}(Q)$, respectively [the last one coincides with the initial topology of $\mathscr{H}(Q)]$. So, let $\eta_{Q}$ be the canonical continuous linear extension of ${ }^{t} j^{2} \odot$ of $E^{\prime} \hat{\odot} E^{\prime}$ into $\mathscr{H}(Q) \odot \mathscr{H}(Q)$. Then, by Proposition 2.2, the functional $\varphi$ which is defined for every $t \in E^{\prime} \hat{\odot} E^{\prime}$ by

(3.4) $\varphi(t)=\operatorname{det}\left(1-2 i \eta_{Q}(t)\right)^{-1 / 2} \exp \left\{i\left\langle m,\left(1-2 i \eta_{Q}(t)\right)^{-1} \circ \eta_{Q}(t)(m)\right\rangle \mathscr{H}_{(Q)}\right\}$

is the Fourier transform of a unique probability on the S.M.V.S. $(E \odot E, \mathscr{B}(E \odot E$, $\left.\left.E^{\prime} \odot E^{\prime}\right)\right)$. But, by (3.3), this probability is nothing else but $\chi_{E}\left(N_{E}(m, Q)\right)$, which proves the first part of the proposition.

The second part is obvious since (3.2) and (3.4) characterize the same distribution on $(E \odot E, \mathscr{B}(E \odot E))$, that is, the one of the random variable $X \otimes X$ when $X$ have the $N_{E}(m, Q)$ distribution.
Now, the following generalization of the Wishart-Bartlett theorem is immediate (see [15]).

Proposirron 3.2. With the assumptions of Proposition 3.1, let $X_{1}, \ldots, X_{n}$ be independent random variables in $(E, \mathscr{B}(E))$ with common distribution $N_{E}(m, Q)$. Then the random variables $\bar{X}=\frac{1}{n} \sum_{i=1}^{n} X_{i}$ and $S=\sum_{i=1}^{n}\left(X_{i}-\bar{X}\right) \otimes\left(X_{i}-\bar{X}\right)$ are independent with distributions $N_{E}(m, Q / n)$ and $W_{E}(n-1,0, Q)$, respectively.

\section{Quadratic analysis of certain Gaussian processes}

This section deals with the derivation of the characteristic function of quadratic forms of certain real Gaussian processes.

Proposition 4.1. Let $T$ be a $\sigma$-compact metric space and let $\{X(t), t \in T\}$ be a real Gaussian process with a.s.-continuous sample paths on $T$ such that:

(i) $E(X(t))=m(t), t \in T$,

(ii) $E(X(s) X(t))-m(s) m(t)=k(s, t),(s, t) \in T \times T$,

(iii) the covariance function $k$ is continuous on $T \times T$

(iv) the mean function $m$ belongs to the R.K.H.S. $\mathscr{H}(k)$;

then, for every regular Borel measure $v$ with compact support on $T \times T$ such that $\nu(A \times B)=v(B \times A)$ for all Borel subsets $A, B$ of $T$, the characteristic function of the r.r.v.: $Z_{p}=\iint_{T \times T} X(s) X(t) d v(s, t)$ is given by:

(4.1) $\quad \tau \in \boldsymbol{R}, \quad \varphi_{Z_{\nu}}(\tau)=\operatorname{det}\left(\mathbf{1}-2 i \tau A_{\nu}^{k}\right)^{-1 / 2} \exp \left\{i \tau\left\langle m,\left(\mathbf{1}-2 i \tau A_{\nu}^{k}\right)^{-1} \circ \boldsymbol{A}_{\nu}^{k}(m)\right\rangle_{\mathscr{H}}(k)_{c}\right\}$ where $A_{v}^{k}$ is the self-adjoint nuclear operator in $\mathscr{H}(k)$ defined by:

$$
f \in \mathscr{H}(k), \quad A_{\nu}^{k}(f)(\cdot)=\iint_{T \times T} f(s) k(t, \cdot) d v(s, t) ;
$$

and

$$
E\left(Z_{v}\right)=\left\langle m, A_{\nu}^{k}(m)\right\rangle_{\mathscr{P}(k)}+\operatorname{Trace}\left(A_{\nu}^{k}\right)=\iint_{T \times T}[m(s) m(t)+k(s, t)] d \nu(s, t) .
$$

As a particular case, for every regular Borel measure $\mu$ with compact support on $T$, the characteristic function of the r.r.v.: $Y_{\mu}=\int_{T} X^{2}(t) d \mu(t)$ is given by:

(4.3) $t \in R, \quad \varphi_{Y_{\mu}}(\tau)=\operatorname{det}\left(1-2 i \tau B_{\mu}^{k}\right)^{-1 / 2} \exp \left\{i \tau\left\langle m,\left(1-2 i \tau B_{\mu}^{k}\right)^{-1} \circ B_{\mu}^{k}(m)\right\rangle_{\mathscr{H}}(k)_{c}\right\}$ where $B_{\mu}^{k}$ is the self-adjoint nuclear operator in $\mathscr{H}(k)$ defined by:

and

$$
f \in \mathscr{H}(k), \quad B_{\mu}^{k}(f)(\cdot)=\int_{T} f(t) k(t, \cdot) d \mu(t)
$$

$$
E\left(Y_{\mu}\right)=\left\langle m, B_{\mu}^{k}(m)\right\rangle_{\mathscr{H}}(k)+\operatorname{Trace}\left(B_{\mu}^{k}\right)=\int_{T}\left[m^{2}(t)+k(t, t)\right] d \mu(t) .
$$


Proof. Let $\{X(t), t \in T\}$ be defined on an arbitrary probability space $(\Omega, \mathscr{A}, P)$. It is easily seen that: $\omega \mapsto X(\omega)=X(\cdot, \omega), \omega \in \Omega$ defines a random variable $X$ taking on its values in the S.M.V.S. $(C(T), \mathscr{B}(C(T)))$, where $C(T)$ denotes the separable Fréchet space of all real continuous functions on $T$ (for the topology of uniform convergence on compact subsets of $T$ ), whose topological dual is identified with the space $M_{C}(T)$ of all regular Borel measures with compact support on $T$, the canonical duality $\left\langle C(T), M_{C}(T)\right\rangle$ being defined by the bilinear form (see [8]):

$$
\langle x, \mu\rangle=\int_{T} x(t) d \mu(t), \quad x \in C(T), \mu \in M_{C}(T) .
$$

Moreover, the distribution $P \circ X^{-1}$ of $X$ is $N_{C(T)}\left(m, Q_{k}\right)$ where $Q_{k}$ is the non-negative quadratic form on $M_{C}(T)$ defined by

$$
Q_{k}(\mu)=\iint_{T \times T} k(s, t) d \mu(s) d \mu(t), \quad \mu \in M_{C}(T) .
$$

So that, $\varphi_{X}(\mu)=E\left(e^{i\langle X, \mu\rangle}\right)=\exp \left\{i\langle m, \mu\rangle-\frac{1}{2} Q_{k}(\mu)\right\}, \quad \mu \in M_{C}(T)$ and $\mathscr{H}\left(Q_{k}\right)$ $\equiv \mathscr{H}(k)$ (see [8])

So, within the framework of Proposition 3.1, the characteristic functional of $X \otimes X$ is determined for all $\nu \in M_{C}(T) \odot M_{C}(T)$ by:

and

$$
\varphi_{X \otimes X}(v)=E\left(e^{\langle\langle X \otimes X, v\rangle\rangle)}=E\left(\exp \left\{i \iint_{T \times T} X(s) X(t) d v(s, t)\right\}\right)\right.
$$

$\varphi_{X \otimes X}(v)=\operatorname{det}\left(\mathbf{1}-2 i \cdot{ }^{t} j^{2 \odot}(v)\right)^{-1 / 2} \exp \left\{i\left\langle m,\left(1-2 i \cdot{ }^{t} j^{2} \odot(v)\right)^{-1} \circ^{t} j^{2 \odot}(\nu)(m)\right\rangle_{\mathscr{H}}(k)_{c}\right\}$.

It is now necessary to explicit the linear mapping ${ }^{t} j^{2 \odot}$ of $M_{C}(T) \odot M_{C}(T)$ into $\mathscr{H}(k) \odot \mathscr{H}(k)$ and then to show that it admits a continuous linear extension of the space $M_{C}^{*}(T \times T)$ of symmetric regular Borel measures with compact support on $T \times T$ into the space $\mathscr{H}(k) \hat{\odot} \mathscr{H}(k)$ of self-adjoint nuclear operators in $\mathscr{H}(k)$. It is easily seen by density arguments that this extension ${ }^{t} j^{2} \hat{\odot}$ is such that for all $\nu$ $\in M_{C}^{*}(T \times T),{ }^{t}{ }^{2} \hat{\odot}(\nu)$ is the operator $A_{v}^{k}$ explicited in (4.2).

Thus, by the results of Section 2 , the characteristic functional of $X \otimes X$ is such that for $\nu \in M_{c}^{*}(T \times T)$,

$$
\varphi_{X \otimes X}(v)=\operatorname{det}\left(\mathbf{1}-2 i A_{v}^{k}\right)^{-1 / 2} \exp \left\{i\left\langle m,\left(1-2 i A_{\nu}^{k}\right)^{-1} \circ A_{\nu}^{k}(m)\right\rangle_{. \mathscr{K}}(k)_{a}\right\} .
$$

So that the characteristic function of the r.r.v.

$Z_{\nu}=\langle\langle X \otimes X, \nu\rangle\rangle=\iint_{T \times T} X(s) X(t) d \nu(s, t)$ is given by $p_{Z_{\nu}}(\tau)=p_{X \otimes X}(\tau \nu), \tau \in \boldsymbol{R}$, which leads to formula $(4,1)$.

The mean of $Z_{\nu}$, is deduced from an obvious extension of the corresponding results in the finite dimensional case (Lemma 2.3).

Now, as a particular case of this theory, we can deduce the characteristic functional of the random variable $G=X^{2}(\cdot)$ with values in $(C(T), \mathscr{B}(C(T)))$ which is defined for $\mu \in M_{C}(T)$ by

$$
\varphi_{G}(\mu)=E\left(e^{i\langle G, \mu\rangle}\right)=E\left(\exp \left\{i \int_{T} X^{2}(t) d \mu(t)\right\}\right) .
$$

Indeed, let $\delta$ be the measurable mapping of $(T, \mathscr{B}(T))$ into $(T \times T, \mathscr{B}(T \times T))$ defined by: $\delta(t)=(t, t), t \in T$.

Then,

$$
\int_{T} X^{2}(t) d \mu(t)=\iint_{T \times T} X(s) X(t) d\left(\mu \circ \delta^{-1}\right)(s, t)
$$

and,

$$
\varphi_{G}(\mu)=\varphi_{X \otimes X}\left(\mu \circ \delta^{-1}\right), \quad \mu \in M_{C}(T) .
$$

It is now easily seen that $A_{\mu \circ \delta^{-1}}^{k}$ takes the form $B_{\mu}^{k}$ given by (4.4) from which we deduce formula (4.3).

This proposition unifies and generalizes the known results about quadratic forms of Gaussian processes related to Brownian motion in the central case. Moreover, the derivation of the characteristic function of these quadratic forms for a wide class of non-central Gaussian processes, constitutes a progress which allows us to look for statistical applications in two differents fields.

\section{Statistical applications}

(a) The classical theory of goodness of fit tests

After the work of Anderson and Darling [1], several authors have used a similar procedure to that of Cramér-Von Mises for various hypotheses testing problems and the bibliography in this domain is large.

Briefly, for testing some hypothesis $H_{0}$ on the basis of an $n$-sample of a random variable they use a criterion which is based on some real statistic $T_{n}$, and then, when investigating the asymptotic properties of the test under $H_{0}$ as $n \rightarrow \infty$, they show that $T_{n}$ converges in distribution to a r.r.v. of the form $Z=\int_{0}^{1} X^{2}(t) \psi(t) d t$ where $\{X(t) ; t \in[0,1]\}$ is a zero-mean real Gaussian process with known covariance function and $\psi$ is some fixed measurable positive function on $[0,1]$ verifying others conditions, as the case may be. Moreover, it can be shown that the asymptotic power of the test under some alternative $H^{\prime}$ in $H_{0}^{c}$ envolves a r.r.v. of the form $Z^{\prime}=\int_{0}^{1}[X(t)+\delta(t)]^{2} \psi(t) d t$ where $\delta$ is some given function on $[0,1]$ (see e.g. [2]).

So, the results of Section 4 may be of some utility when deriving the distribution of the r.r.v. $Z$ and $Z^{\prime}$, for several choices of the functions $\psi$ and $\delta$ and thus, they may provide some contribution to the asymptotic study of these tests.

Let us quote as examples of applications among the recent literature on the subject:

- the goodness of fit tests when nuisance parameters are estimated from the data (see [3]); 
- testing a sequence of observation from exponential-type distribution for no change of parameter at unknown time (see [6]);

- testing symmetry with a Cramér-Von Mises type statistic (see [10]);

- etc.

(b) Test of significance of the mean function of a Gaussian process with known covariance function

Results of Section 4, especially formula (4.3), allow us to propound a class of tests of significance of the mean function of certain types of Gaussian processes basec on a quadratic criterion, the power function of which are entirely explicited.

Actually, we are concerned with the problem of testing the hypothesis " $m$ $=m_{0}$ " against " $m \neq m_{0}$ " in the C.E.T. Gaussian statistical space:

$$
\left(C(T), \mathscr{B}(C(T)),\left\{N_{C(T)}\left(m ; Q_{k}\right) ; m \in \mathscr{H}(k)\right\}\right)
$$

all terms of which are defined in Section $4 ; m_{0}$ being a fixed function in $\mathscr{H}(k)$

Without loss of generality we can assume that $m_{0}=0$.

Definition 5.1. A quadratic test of " $m=0$ " against " $m \neq 0$ ", in the statistical space (5.1), will be any test of the form

$$
\Phi_{\mu, l}(x)=\left\{\begin{array}{lll}
1 & \text { if } \quad\left[\int_{T} x^{2}(t) d \mu(t)\right]^{1 / 2}>l, \\
0 & \text { if } \quad\left[\int_{T} x^{2}(t) d \mu(t)\right]^{1 / 2}<l,
\end{array} \quad x \in C(T),\right.
$$

for some non-negative measure $\mu \in M_{C}(T)$ and some positive real number $l$.

Proposition 5.1. For every $\alpha, 0 \leqslant \alpha \leqslant 1$ there exists an unbiased quadratic test of size $\alpha$ for testing " $m=0$ " against " $m \neq 0$ ".

Proof. Taking for $\mu$ the Dirac measure $\delta_{t_{0}}$ at some fixed point $t_{0} \in T$, the test $\Phi_{\delta_{t_{0}, l}}$ reduces to $\mathbf{1}_{\left\{\left|x\left(t_{0}\right)\right| \geqslant l\right\}}(x) ; x \in C(T)$. It is similar unbiased for testing the linear hypothesis $H_{t_{0}}$ : " $m\left(t_{0}\right)=0$ " against " $m\left(t_{0}\right) \neq 0$ ", for, it coincides with the UMPB test of the simple hypothesis " $m\left(t_{0}\right)=0$ " against " $m\left(t_{0}\right) \neq 0$ " in the image statistical space of (5.1) by the real linear statistic: $x \mapsto\left\langle x, \delta_{t_{0}}\right\rangle$, which is the scalar Gaussian statistical space

$$
\left(\boldsymbol{R}, \mathscr{B}(\boldsymbol{R}),\left\{N\left(m\left(t_{0}\right), k\left(t_{0}, t_{0}\right)\right) ; m\left(t_{0}\right) \in \boldsymbol{R}\right\}\right) .
$$

Thus, for all $m \in \mathscr{H}(k)$, the power function of the test $\Phi_{\delta_{t_{0}, l\left(t_{0}, \alpha\right)}}$ of size $\alpha$ is such that:

as it is easily shown

$$
\beta_{\Phi_{\delta_{t_{0}}, l\left(t_{0}, \alpha\right)}(m)}\left\{\begin{array}{lll}
=\alpha & \text { if } & m\left(t_{0}\right)=0 \\
>\alpha & \text { if } & m\left(t_{0}\right) \neq 0
\end{array}\right.
$$

Consequently, the test $\Phi_{\delta_{t_{0}} l\left(t_{0}, \alpha\right)}$ is unbiased of size $\alpha$ for testing " $m=0$ " against " $m \neq 0$ " since the value of its power function at any $m \neq 0$ in $\mathscr{H}(k)$ is $\geqslant \alpha$ according as $m\left(t_{0}\right) \neq 0$ or $m\left(t_{0}\right)=0$.
Now, let $\mu$ be such that $\int_{-\infty}^{+\infty}\left|\varphi_{Y \mu}^{m}(\tau)\right| d \tau<\infty$ where $\varphi_{Y_{\mu}}^{m}$ is given by (4.3) when the value of the parameter is $m$. Then, by a classical theorem on characteristic functions, the d.f. $F_{Y_{\mu}}^{m}$ of $Y_{\mu}$ is everywhere derivable and it is given by:

$$
F_{Y_{\mu}}^{m}(u)=\frac{1}{2 \pi} \int_{-\infty}^{+\infty} \frac{e^{-i \tau u}-1}{-i \tau} \varphi_{Y_{\mu}}^{m}(\tau) d \tau
$$

so that the power function of the quadratic test $\Phi_{\mu, l}$ is given for every $m \in \mathscr{H}(k)$ by:

$$
\beta_{\Phi_{\mu, l}}(m)=1-\frac{1}{2 \pi} \int_{-\infty}^{+\infty} \frac{e^{-i \tau l 2}-1}{-i \tau} \varphi_{Y \mu}^{m}(\tau) d \tau .
$$

In particular, the significance level of the test is given by:

$$
\alpha=\beta_{\Phi_{\mu, l}}(0)=1-\frac{1}{2 \pi} \int_{-\infty}^{+\infty} \frac{e^{-i \tau l^{2}}-1}{-i \tau} \operatorname{det}\left(1-2 i \tau B_{\mu}^{k}\right)^{-1 / 2} d \tau
$$

which theoretically gives $l=l(\alpha, \mu)$

PROPOSITION 5.2. For fixed $l$ and $\mu$, the power function $m \mapsto \beta_{\Phi_{\mu, l}}(m)$ is continuous on $\mathscr{H}(k)$. For fixed $l$ and $m$, the functional $\mu \rightarrow \beta_{\Phi_{\mu, l}}(m)$ is continuous on the positive cone $M_{C}^{+}(T)$ endowed with its vague topology.

These elementary results incite one to carry on the theory of quadratic tests especially in the direction of the optimization of the choice of $\mu$ when $T$ is compact, with respect to some criterion to be defined. Moreover, the study of the provided confidence regions may be an interesting field of investigation.

Finally, quadratic criteria may be used to derive tests of equality of mean functions based on observed samples $x$ and $x^{\prime}$ of two independent Gaussian processes $X$ and $X^{\prime}$ with same known covariance function. Indeed, by similar arguments to that used above, any test of the form

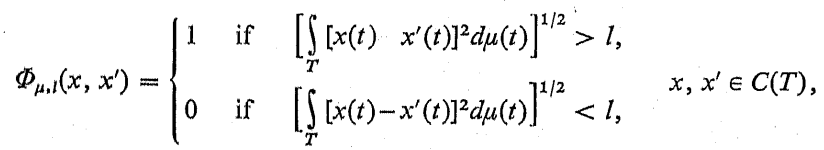

will be similar for testing " $m=m^{\prime}$ " against " $m \neq m^{\prime \prime}$ " in the product statistical space:

$\left(C(T), \mathscr{B}(C(T)),\left\{N_{C(T)}\left(m, Q_{k}\right), m \in \mathscr{H}(k)\right\}\right) \cdot\left(C(T), \mathscr{B}(C(T)),\left\{N_{C(T)}\left(m^{\prime}, Q_{k}\right) ;\right.\right.$

$$
\left.\left.m^{\prime} \in \mathscr{H}(k)\right\}\right)
$$

since $X-X^{\prime} \sim N_{C(T)}\left(m-m^{\prime}, Q_{2 k}\right)$.

This theory and several applications will be considered in subsequent studies. 


\section{References}

[1] T. W. Anderson and D. A. Darling, Asymptotic theory of certain goodness of fit criteria based on stochastic processes, Ann. Math. Stat. 23, $\mathrm{n}^{\circ} 2$ (1965), pp. 192-212.

[2] D. M. C $\mathrm{h}$ i b i s o v, An investigation of the asymptotic power of the tests of fit, Theor. Prob. \& Appl. vol. X, $\mathrm{n}^{\circ} 3$ (1965), pp. 421-437.

[3] J. $\mathrm{D}$ u $\mathrm{r} \mathrm{b}$ i $\mathrm{n}$, Weak convergence of the sample distribution function when parameters are estimated, Ann. Stat. 1, $\mathrm{n}^{\circ} 2$ (1973), pp. 279-290.

[4] A. G r o th en di e ck, La théorie de Fredholm, Bull. Soc. Math. France 84 (1956), pp. 319384.

[6] $\mathrm{X}$. B. $\mathrm{M}$ a c $\mathrm{N}$ e i11, Tests for change of parameter at unknown times and distributions of some related functionals of Brownian motion, Ann. Stat. 2, $\mathrm{n}^{\circ} 5$ (1974), pp. 950-962.

[7] J. N e v e u, Processus aléatoires gaussiens, Séminaire de Mathématiques Supérieures, Montréal 1968.

[8] B. S. R a j p u t, On Gaussian measures on certain locally convex spaces, Journ. Multivariate Analysis 2 (1972), pp. 282-306.

[9] J u. A. R o z a n o v, Infinite-dimensional Gaussian distributions, Amer. Math. Soc., Providence 1971.

[10] E. D. R o th $\mathrm{m}$ a n and M. W o o d r o o f e, A Cramér-Von Mises type statistic for testing symmetry, Ann. Math. Stat. 43, no 6 (1972), pp. 2035-2038.

[11] H. H. S c h a e f e r, Topological vector spaces, Springer V., 1970

[12] J. L. S o l e r, m-dual d'un espace vectoriel mesurable et structures statistigues de type exponentiel, C. R. Acad. Sc., Paris, 277, Ser. A (1973), pp. 49-52.

[13] -, Infinite-dimensional exponential type statistical spaces (Generalized exponential families) European Meeting of Statisticians, Grenoble, 1976, North-Holland.

[14] —, La loi de probabilité de Wishart associée à un élément aléatoire gaussien décentré d valeurs dans un espace de Fréchet séparable. Application aux fonctions aléatoires gaussiennes, C. R. Acad. Sc., Paris, 281, Ser. A (1975), pp. 471-474.

[15] -, Introduction aux structures statistiques infinidimensionnelles, To appear, Univ. Scientifique et Médicale de Grenoble.

Presented to the semester MATHEMATICAL STATISTICS

September 15-December 18,1976
BANACH CENTER PUBLICATIONS, VOLUME 6 PWN-POLISH SCIENTIFIC PUBLISHERS

\section{A SIMPLE TECHNIQUE FOR PROVING UNBIASEDNESS OF TESTS AND CONFIDENCE REGIONS}

EMIL SPJøTVOLL

Department of Mathematics and Statistics, Agricultural University of Norway, Norway

\section{Introduction}

Let $X$ be a random variable with probability density $f(x, \theta)$. The parameter $\theta$ belongs to a set $\Omega$. The present author [5] studied confidence sets based upon the likelihood function. The sets are of the form

$$
S(x)=\left\{\theta: \frac{f(x, \theta)}{\sup _{\theta} f(x, \theta)} \geqslant c\right\},
$$

where $c$ is the largest $c$ such that

$$
P_{\theta}\{\theta \in S(X)\} \geqslant 1-\alpha .
$$

From the confidence sets we can also easily derive a test of the hypothesis

$$
H: \theta=\theta_{0} \text { against } \theta \neq \theta_{0} ;
$$

the test rejects if $\theta_{0} \notin S(x)$. Then the probability of a false rejection is

$$
P_{\theta_{0}}\left\{\theta_{0} \notin S(X)\right\} \leqslant \alpha .
$$

The author [5] has shown that under certain assumptions the confidence sets (1) are unbiased. Then the test which rejects $H$ when $\theta_{0} \notin S(x)$ is also unbiased.

In this paper we shall consider the situation where the distribution of $X$ depends upon two parameters $\theta$ and $\eta$, where $\eta$ is a nuisance parameter. Suppose that there exists a statistics $Y(X)$ with density $g(y, \theta)$ (with respect to a measure $\mu$ ) which depends only upon $\theta$ and not upon $\eta$. Then, from the likelihood function $g(y, \theta)$, we can construct confidence sets of the form

$$
T(y)=\left\{\theta: \frac{g(y, \theta)}{\sup _{\theta} g(y, \theta)} \geqslant c\right\} .
$$

Under the following assumptions A1-A3 (see Spjøtvoll [5]) the confidence sets $T(y)$ are unbiased. 